\title{
Effects of fructose not so sweet
}

The role of dietary fructose in causing diseases such as obesity and fatty liver disease has been controversial. Kylie Kavanagh and her team at Wake Forest School of Medicine (Winston-Salem, NC) studied macaques and vervet monkeys who were allowed to eat as much as they wanted of low-fat food with added fructose for seven years, as compared to a control group fed a low-fructose, low-fat diet for the same time period. The high-fructose group gained 50\% more weight and developed diabetes and hepatic steatosis at much higher rates than the control group. Hepatic steatosis is the accumulation of fat in the liver and is an early stage in the development of nonalcoholic fatty liver disease.

In light of these findings, the researchers decided the next step was to determine whether hepatic steatosis developed because of the animals' weight gain or directly because of the higher fructose consumption. The team carried out another experiment, this time designed to prevent weight gain in the high-fructose

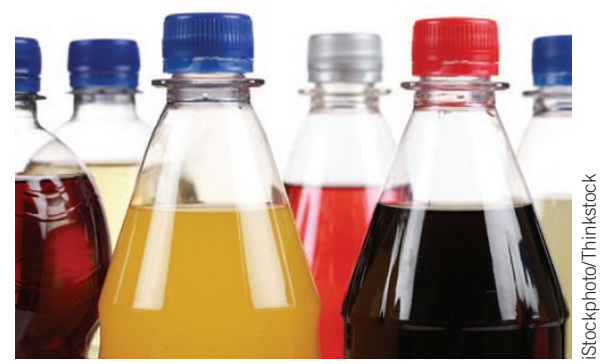

diet group. In this study, both groups were fed calorie-controlled diets, one consisting of $24 \%$ fructose and the other containing only $0.5 \%$ fructose. Though the foods comprising each diet were different, both diets had the same amount of fat, carbohydrates and protein.

After six weeks, the researchers collected blood samples so that they could look for biomarkers of liver damage. Despite maintaining healthy weights, the animals fed the high-fructose diet had more than double the liver damage of the control group (Am. J. Clin. Nutr. published online 19 June 2013; doi:10.3945/ acjn.112.057331). The researchers were surprised by how quickly the liver was affected and how extensive the damage was, especially without weight gain being a factor.

Kavanagh and her team also collected fecal samples and performed intestinal biopsies to determine which bacteria were present in the intestine. Live bacteria or bacterial products present in the gastrointestinal tract are passed to the liver in a process called microbial translocation. In the high-fructose group, intestinal bacteria migrated to the liver more rapidly and caused more damage there, suggesting that the fructose somehow caused the intestines to be more permeable.

"Is a calorie a calorie? Are they all created equal? Based on this study, we would say not," Kavanagh said. The next step will be to test the effects of other sugars, such as dextrose, in addition to fructose to see if they have similar damaging effects on the liver.

\section{Kara Rosania}

\section{TRAVERSING THE RETINA WITHOUT DAMAGE}

Hereditary retinal degeneration, in which mutations in photoreceptors and other delicate cells of the eye's outermost retinal layer lead to deterioration and visual impairment, affects roughly 1 in 3,000 people worldwide. Gene therapy can successfully deliver normal copies of the mutated genes to the affected cells using adeno-associated virus (AAV) but requires that the virus be injected through the retina. These injections reach only a fraction of the affected cells and, moreover, can damage the fragile retina, making them unsuitable for treating the many forms of retinal degeneration in which the retinal tissue is structurally compromised.

A technique recently published in Science Translational Medicine (5, 189ra76; 2013) addresses the resultant need for a less invasive method of delivering gene therapy across the retina. John Flannery and David Schaffer (University of California Berkeley) led the project, engineering a variant of AAV to pass through the retina with its genetic cargo. This variant, $7 \mathrm{~m} 8$, can be injected into the vitreous, the more accessible gel-like fluid that fills the center of the eye, sparing the retina. The genes it carries are expressed throughout the retina and the optic nerve but not outside the eye. Expression of the cargo genes can be further restricted to cells of interest (e.g., photoreceptors) by using a specific promoter to control the activation of $7 \mathrm{~m} 8$.

Flannery and Schaffer's group tested $7 \mathrm{~m} 8$-based gene therapy in mouse models of two forms of inherited retinal degeneration: $X$-linked retinoschisis and Leber congenital amaurosis type 2. In both cases, delivery of wild-type copies of the affected genes using $7 \mathrm{~m} 8$ rescued the disease phenotype and improved the mice's vision on a long-term basis.

The team also evaluated $7 \mathrm{~m} 8$-based gene delivery across the retina in cynomolgus macaques and confirmed its success. The inner layers of the retina are thicker in nonhuman primates than in rodents, presenting a more difficult barrier to trans-retinal gene therapy.

"Building upon 14 years of research, we have now created a virus that you can inject into the liquid vitreous humor inside the eye and it delivers genes to a very difficult-to-reach population of delicate cells in a way that is surgically much less invasive and safer," Schaffer told NIH Research Matters. The technique's success suggests that this approach may also be applicable to developing gene therapy vectors that can cross other barriers: endothelial (such as the blood-brain barrier), intra-organ or tumoral. 